\title{
Serial Digital Color Image Watermarking using Composite Scheme
}

\author{
Dayanand.G.Savakar \\ Department of Computer Science, P.G. Center, Rani Channamma University, Vijaypur-586108. Karnataka, India. \\ Email: dgsavakar@gmail.com.

\section{Anand Ghuli} \\ Department of Computer Applications, B.L.D.E.A's V.P.Dr.P.G.Halakatti College of Engineering and Technology., \\ Vijayapur-586103, Karnataka, Affiliated to Visvesvaraya Technological University, Belagavi., Karnataka, India. \\ Email: anandghuli@gmail.com
}

Received: 01 July 2017; Accepted: 07 August 2017; Published: 08 November 2017

\begin{abstract}
Digital watermarking is one of the ways to have Copyright protection for digital information. The digital watermarking scheme used for watermark embedding has to satisfy robustness property to ensure the security of the secret information hidden. The scheme presented here will support the above said statement significantly. We propose here the scheme as composition of both blind and non-blind digital watermarking technique in a process of serial watermarking. A secret binary image is embedded in the first cover image to get first watermarked image by using blind watermarking technique. Then this first watermarked image is again embedded into second cover image to get serial watermarked image using non-blind watermarking technique. To extract secret binary image, first non-blind watermark extraction technique and then blind watermark extraction techniques are used. From this composite approach and serial watermark embedding procedure, we achieved considerable fidelity and robustness against - Rotation, JPEG compression and for noises Salt \& pepper, Gaussian, Speckle, Poisson and multiple noises.
\end{abstract}

Index Terms-Composite scheme, Serial watermarking, First cover image, Second cover image, Serial watermarked image, Blind, Type-II Non-blind.

\section{INTRODUCTION}

The Digital Image Watermarking is contributing significantly to copyright protection of Digital Image. The inherent characteristics of color image making a wealthy way to embed secret images as watermarks into it. The Digital Image Watermarking is quite a young field and grabbed a lot of attention of researchers. In relatively within a short span of time, significant and notable contributions have been observed in the field of Digital Image Watermarking with respect to theoretical and practical aspects. This potential area has the ability to address many security related issues like, copyright protection, usage monitoring, tracing of distribution and usage control [17][19]. However, in most of the digital watermarking schemas; robustness, fidelity, and capacity are the major properties to be addressed efficiently and convincingly [17][19]. This paper presents the scheme which will be another element in the synergy of contributing in the field of secured digital image watermarking. The watermarked image is an image with the secret watermark embedded into it. Then this watermarked image can be published, and the owner of the information can claim his ownership by retrieving the watermark hidden into it [17]. As a basic requirement, the watermarking scheme should be robust against various types of attacks on the image to be transmitted [17].

\section{RELATED WORK}

Different types of transformations are used or being used with different watermark embedding / retrieving procedure and techniques to attain considerable robustness. In most of the cases discrete wavelet transformations (DWT) are used to decompose the image into the different set of frequency sub-bands like LL, LH, HL, HH to locate suitable area[13][14][18][23]. DWT, The Artificial bee colony and discrete cosine transformation in combination with various schemes used to attain robustness with respect to block based segmentation and others types of segmentation of cover image providing robustness to embed a watermark in accordance with the suitable embedding procedure [5][7][12][13][14]. The usage of fractional Krawtchouk transform (FrKT) in the digital image watermarking has given better robustness by using eigenvalue decomposition [1]. The scale invariant feature transform (SIFT) is used to extract high invariance feature points then by using non-sub sampled contourlet transform (NSCT) the watermark will be embedded using extracted features[2]. Learning based conventional neural network is also used for digital image watermarking, as it needs 
the knowledge of architecture and keys of embedding procedure, the intruder may feel difficulty in extracting watermark[4]. The various meta-heuristic techniques are used to achieve optimized performance [5]. The quaternion discrete Fourier transform used to get the maximum central region of the image and the watermark is embedded using magnitudes of low-frequency information [6]. SVD is used to embed the watermark into singular values of the obtained matrix form cover image [12]. Even the hybrid techniques like least significant bit plane and most significant bit planes are used in connection with DWT, SVD ABC to achieve good robustness and user specific threshold imperceptibility [10][11][12]. A non-blind approach based on DWT-SVD digital watermarking using Alpha Blending and Arnold transformation in $\mathrm{YCbCr}$ Color space is robust on geometric attacks [20]. A chaos based digital image watermarking algorithm based on redundant discrete wavelet transform (RDWT) and singular value decomposition (SVD) is used to achieve robustness [21]. RDWT is shift invariant and it improves the robustness against additive noise because of its redundancy. SVD is a complementary technique for watermarking schemes providing good stability and perceptual invisibility [21]. Instead of DWT, either complex wavelet transform(CWT), Multi wavelet or complex and rotated wavelet transform can be used to make the system more robust and secure [22].

When we observe the work done in this area, we can say that there is a space for contribution in achieving robustness in accordance with fidelity and capacity parameters of digital image watermarking. The complete robustness with respect to Gaussian noise, Salt \& Peppers, Speckle Noise, Poisson Noise, Rotation, JPEG, and Cropping is a task of interest in this work. Here with serial watermarking as a composition of blind and nonblind watermarking technique more robustness and suitable imperceptibility can be achieved. We propose the scheme which uses both blind (key is only required to extract secret image [17]) and Type-II non-blind (TypeII non-blind system require the copy of embedded watermark and cover image for extraction [17]) digital watermarking to attain robustness, fidelity properties of the digital watermarking. The blind digital image watermarking technique uses discrete wavelet transformation (DWT) to locate the suitable area to embed watermark with a predefined block of binary digit sequences. And this image once again embedded into another image using non-blind watermarking technique using DWT and singular value decomposition (SVD)[12][18]. During the retrieval process, first nonblind technique and then blind technique are used. The following discussion includes methodology, results \& discussion, conclusion, and references.

\section{Methodology}

The scheme presented here is composed of two stages; stage-1 and stage-2. The stage-1 uses a blind watermarking technique to embed a secret binary image into a first cover image to get a first watermarked image. This first watermarked image again embedded into second cover image using a non-blind technique to get serial watermarked image. This whole process is depicted in Fig.1.

\section{A. Composite scheme for serial watermark embedding}

The composite scheme for serial watermark embedding uses the 2 level (Haar) Discrete Wavelet Transformation (DWT), $4 \times 4$ binary sequence block and Singular Value Decomposition (SVD). The terms used in proposed scheme are i) First Cover Image - The color image used to embed the secret binary image. ii) Secret binary image - watermark to be embedded into the first cover image. iii) First Watermarked Image - The color image embedded with a secret binary image. iv) Second Cover Image - The color image used to embed the First Watermarked Image. v) Serial Watermarked Image - The Second Cover Image embedded with First Watermarked Image. The following sections 2.1.1 to 2.1.3 describes the tools - DWT, Binary sequence block, SVD respectively and 2.1.4 to 2.1.5 describes how the tools are used in stage- 1 and stage- 2 of proposed scheme respectively.

\section{Al Discrete Wavelet Transformation (DWT)}

The blind watermarking process of stage-1 uses DWT (Haar) to extract HL bands because HL bands will have very clear horizontal edges and are useful for locating the suitable area to embed secret binary image [14]. DWT decomposition will split the input signal into highfrequency and low-frequency parts. To analyze the high frequencies and low frequencies, the signal is passed through a series of high pass and low-pass filters respectively with different cutoff frequencies at different resolutions. To represent the DWT decomposition of a signal, consider the one-dimensional signal $x[n]$ spanned in a frequency band of 0 to $\pi$ radians. The signal $x[n]$ is first passed through a high-pass filter $\mathrm{g}[\mathrm{n}]$ and a low-pass filter $\mathrm{h}[\mathrm{n}]$. According to the Nyquist's rule the half of the samples are eliminated, now the signal has the highest frequency of $\pi / 2$ radians. The signal is sub sampled by 2 by discarding every second sample. This gives one level of decomposition of $\mathrm{x}[\mathrm{n}]$ and expressed mathematically as equation (1) and (2).

$$
\begin{aligned}
& y_{\text {high }}[k]=\sum_{n} x[k] g[2 k-n] \\
& y_{\text {low }}[k]=\sum_{n} x[k] h[2 k-n]
\end{aligned}
$$

where $y_{\text {high }}[k]$ and $y_{\text {low }}[k]$ are the outputs of the respective high-pass and low-pass filters after subsampled by 2 . 


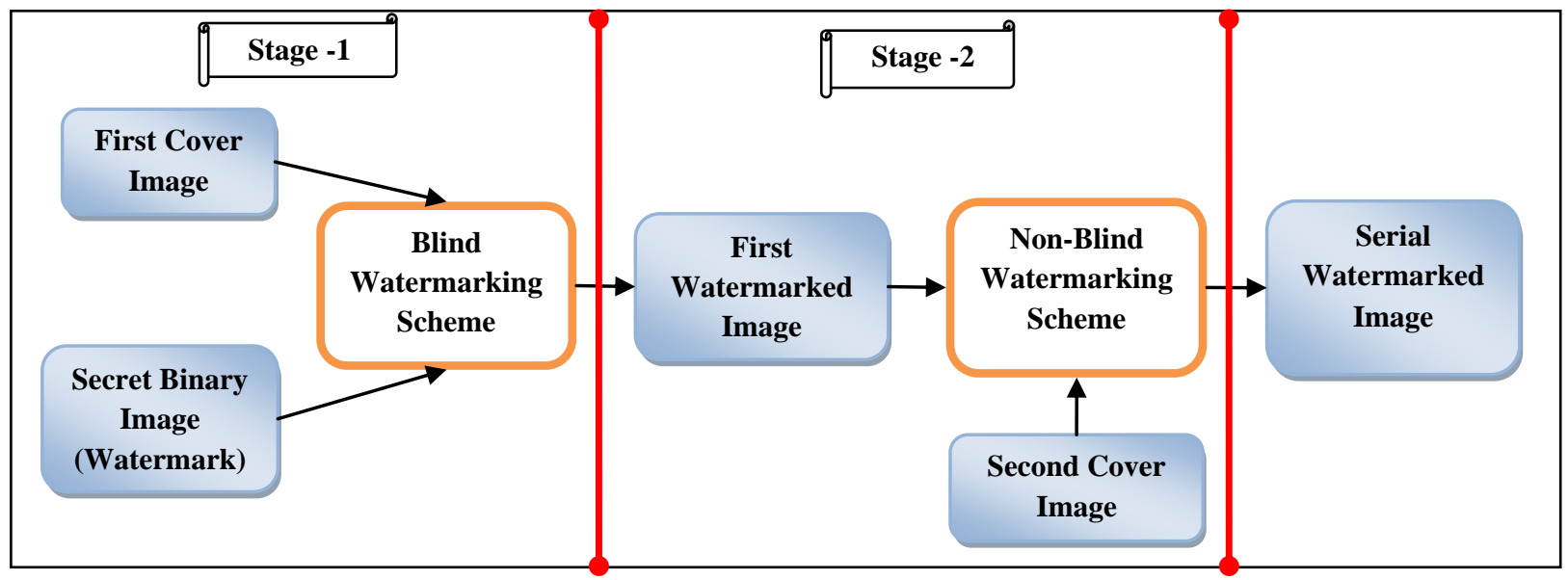

Fig.1. Composite scheme for serial Watermarking Process

The above process can be repeated for further decomposition. The outputs of the high-pass and lowpass filters are called DWT coefficients and by these DWT coefficients, the signal can be easily reconstructed using the Inverse Discrete Wavelet Transform (IDWT). In Inverse Discrete Wavelet Transform (IDWT) the above process is followed in reverse order for the reconstruction. The signals at every level are upsampled by two and passed through the synthesis filters $\boldsymbol{g}^{\prime}[\boldsymbol{n}]$ and $\boldsymbol{h}^{\prime}[\boldsymbol{n}]$ (high-pass and low-pass, respectively) and added to produce the original signal. Mathematically IDWT can be expressed as follows:

$$
x[n]=\sum y_{\text {high }}[k] g[-n+2 k]+y_{\text {low }}[k] h[-n+2 k]
$$

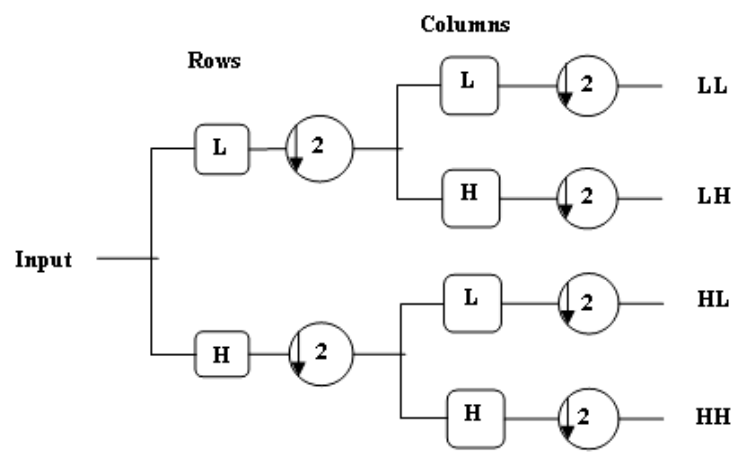

Fig.2. Wavelet Decomposition

\begin{tabular}{|c|c|c|}
\hline \multirow{2}{*}{$L L$} & $L L$ & $H L$ \\
\cline { 2 - 3 } & $H L$ & $H H$ \\
\hline \multirow{2}{*}{$L H$} & \multicolumn{2}{|c|}{$H H$} \\
\hline
\end{tabular}

\begin{tabular}{|c|c|c|}
\hline$L L$ & $H L$ & \multirow{2}{*}{$H L$} \\
\cline { 1 - 1 }$H L$ & $H H$ & \\
\hline \multicolumn{2}{|c|}{$L H$} & \\
& $H H$ \\
\hline
\end{tabular}

Fig.3. Wavelet Sub-band Representation

In an image processing application, the twodimensional wavelet is used. This will limit to only square images with the height and width equal to the power of two. If the image is of the size $\mathrm{N} \times \mathrm{N}$ then $\mathrm{N}=$ $2^{\text {n }}$. Fig. 2 represents one level DWT decomposition of an image. The wavelet analysis is the application of highpass and low-pass filters that separate the original image into four sub-images with the different frequency components. For an image of size $\mathrm{N} \times \mathrm{N}$ wavelet decomposition generates $\mathrm{N}^{2}$ wavelet coefficients. Fig.3 shows the steps used for one-level decomposition of an image. Initially, the image is passed through the highpass and low-pass filters along the rows and the results of each filter are down-sampled by two. The two subsignals correspond to the high-pass and low-pass components along the rows are again passed through high-pass and low-pass filtered along the column. The results are again down-sampled by two. In this way, the original image is split into four sub-images each of size $\mathrm{N} / 2 \times \mathrm{N} / 2$ which contains the different frequency components. This composition of frequency components is known as the inverse decomposition. Fig.4 represents one level inverse DWT.

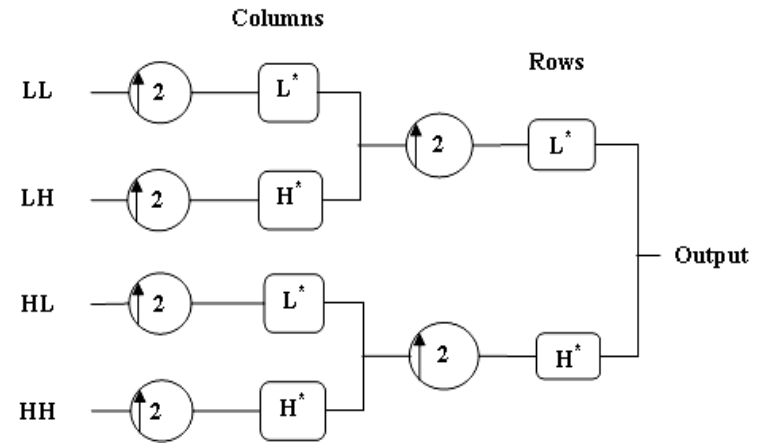

Fig.4. One level Inverse DWT

The two-dimensional wavelet transform can be seen as one-dimensional wavelet transform along the $\mathrm{x}$ and $\mathrm{y}$ axes. Mathematically the wavelet transform is convolution operation, which is equivalent to pass the pixel values of an image through a low-pass and highpass filters. The wavelet transform of an image decomposes the image into four frequency bands, namely, the LL1, HL1, LH1 and HH1 bands. H and L denote the high-pass and low-pass filters respectively. The approximated image LL is obtained by low-pass filtering in both row and column directions. The detailed images, 
LH, HL and HH contain the high- frequency components. To obtain the next coarse level of wavelet coefficients the subband LL1 is further decomposed and critically sampled. Similarly, LL2 will be used to obtain further decomposition. By decomposing, the approximated image at each level into four sub-images forms the pyramidal image tree. This results in two-level wavelet decomposition of the image.

\section{A2 Pre-Processing of Secret Binary Image}

The secret binary image is converted into row matrix of sequences of $0 \mathrm{~s}$ and $1 \mathrm{~s}$ called secret binary image vector. To process HL2 sub-band block wise, the block structure is framed as shown in Fig.5. Now uniformly distributed random numbers are generated with the help of key image of size $1 \times 35$. Separate sequences of pseudo random numbers are formulated for $0 \mathrm{~s}$ and $1 \mathrm{~s}$ respectively known as pn_sequence_0 and pn_sequence_1.

\begin{tabular}{|l|l|l|l|}
\hline 0 & 1 & 0 & 1 \\
\hline 1 & 0 & 1 & 0 \\
\hline 0 & 1 & 0 & 1 \\
\hline 1 & 0 & 1 & 0 \\
\hline
\end{tabular}

Fig.5. Sample of $4 \times 4$ Binary Sequence block used (For watermark insertion and extraction)

\section{A3 Singular Value Decomposition (SVD)}

The singular value decomposition [12][18] of a $\mathrm{m} \times \mathrm{n}$ real or complex matrix $\mathrm{A}$ is a factorization of the form

$$
\mathrm{A}_{M x N}=\mathrm{U}_{M x M} \mathrm{~S}_{M x N} \mathrm{~V}^{\mathrm{T}}{ }_{N x N}
$$

Where

$\mathbf{U}^{\mathrm{T}} \mathbf{U}=\mathbf{I}_{\mathrm{MxM}}$

$\mathbf{V}^{\mathrm{T}} \mathbf{V}=\mathbf{I}_{\mathrm{NxN}}$ (i.e. $\mathrm{U}$ and $\mathrm{V}$ are orthogonal)

Where $\mathrm{U}$ is a $\mathrm{m} \times \mathrm{m}$ real or complex unitary matrix, $\mathrm{S}$ is a $\mathrm{m} \times \mathrm{n}$ diagonal matrix with nonnegative real numbers on the diagonal, and $\mathrm{V}^{\mathrm{T}}$ is the conjugate transpose of $\mathrm{V}$ with a $\mathrm{n} \times \mathrm{n}$ real or complex unitary matrix. The diagonal entries $S$ are known as the singular values of $A$. The $m$ columns of $U$ and the $n$ columns of $V$ are called the left singular vectors and right singular vectors of $\mathrm{A}$, respectively. In singular value decomposition of an image, each singular value represents the luminance of image layer and the corresponding pair of the singular vector represents the geometry of the image layer [12][18]. The singular values in a digital image are less affected by general image processing operations because the bigger singular values preserve most energy of an image and resist against attacks. The singular values possess intrinsic algebraic image properties. In SVD-based image watermarking, several approaches are possible. A common method is to apply SVD to the entire cover image and to modify all the singular values to embed the watermark. But by applying SVD on DWT transformed sub-band images will improve robustness, because the important property of SVD based watermarking is that the modified singular values of an image will change by very small values for different types of attacks [12] [18]. The contents of these matrices are computationally stable and hence these are used in connection with DWT for more robustness.

\section{A4 Stage-1 Watermark Embedding Process (Blind)}

Fig.6 demonstrates Stage-1 of the composite watermarking scheme; the two level Haar wavelet decomposition is used on the input image to get four nonoverlapping multi-resolution sub-bands. To achieve acceptable performance with respect to imperceptibility and robustness it is found suitable to embed the watermark in the middle frequency sub-bands LHx and HLx [11]. Two level decomposed HL2 sub-band is considered for embedding the secret binary image into it by $4 \times 4$ block processing technique [14]. The inverse DWT is applied to reconstruct the original image with the secret binary image embedded into it [16].HL2 sub-band is scanned from left to right with the help of $4 \times 4$ binary sequence block. If secret binary image vector is 0 and content of the $4 \times 4$ binary sequence block is 1 then a constant gain factor is multiplied with pn_sequence_0 and added with the under scanning content of $4 \times 4$ HL2 block. If secret binary image vector is 1 and content of the $4 \times 4$ binary sequence block is 1 then a constant gain factor is multiplied with pn_sequence_1 and added with the under scanning content of $4 \times 4$ HL2 block. This whole process is depicted in Fig.6 and in Step. 1 to Step.7 of Algorithm-1.

\section{A5 Stage-2 Watermark Embedding Process (Non-Blind)}

Stage-2 employs the non-blind watermarking technique, where the outcome of stage- 1 is taken i.e first watermarked image to embed into second cover image. Here Haar single level DWT is applied on a second cover image to extract lower frequency LL sub-band where most of the image information (energy) is stored [14][16]

As the LL sub-band contains a lot of image information embedding the watermark may degrade the image significantly. So this LL sub-band image is divided into red $(\mathrm{R})$, green $(\mathrm{G})$ and blue $(\mathrm{B})$ channels and singular value decomposition (SVD) is applied to each component to get computationally sustainable components as $\mathrm{U}, \mathrm{S}$ and $\mathrm{V}$ matrices of the transformed image. The Same process is applied for the second cover image also.

Fig.7 demonstrates Stage-2 watermarking scheme. The combining function shown in the Fig.7 is framed such that S components of DWT applied LL sub-bands of the second cover image and first watermarked image are added with each $\mathrm{R}, \mathrm{G}$ and $\mathrm{B}$ channel components multiplied with a gain factor. Later same components are multiplied with other $\mathrm{U}$ and $\mathrm{V}$ values of $\mathrm{R}, \mathrm{G}$ and $\mathrm{B}$ 
values and concatenate them to get watermarked image. Then apply inverse DWT to get serial watermarked image. The detailed process is presented in Algorithm 1.
This combined approach increases the robustness and also satisfies the fidelity criteria.

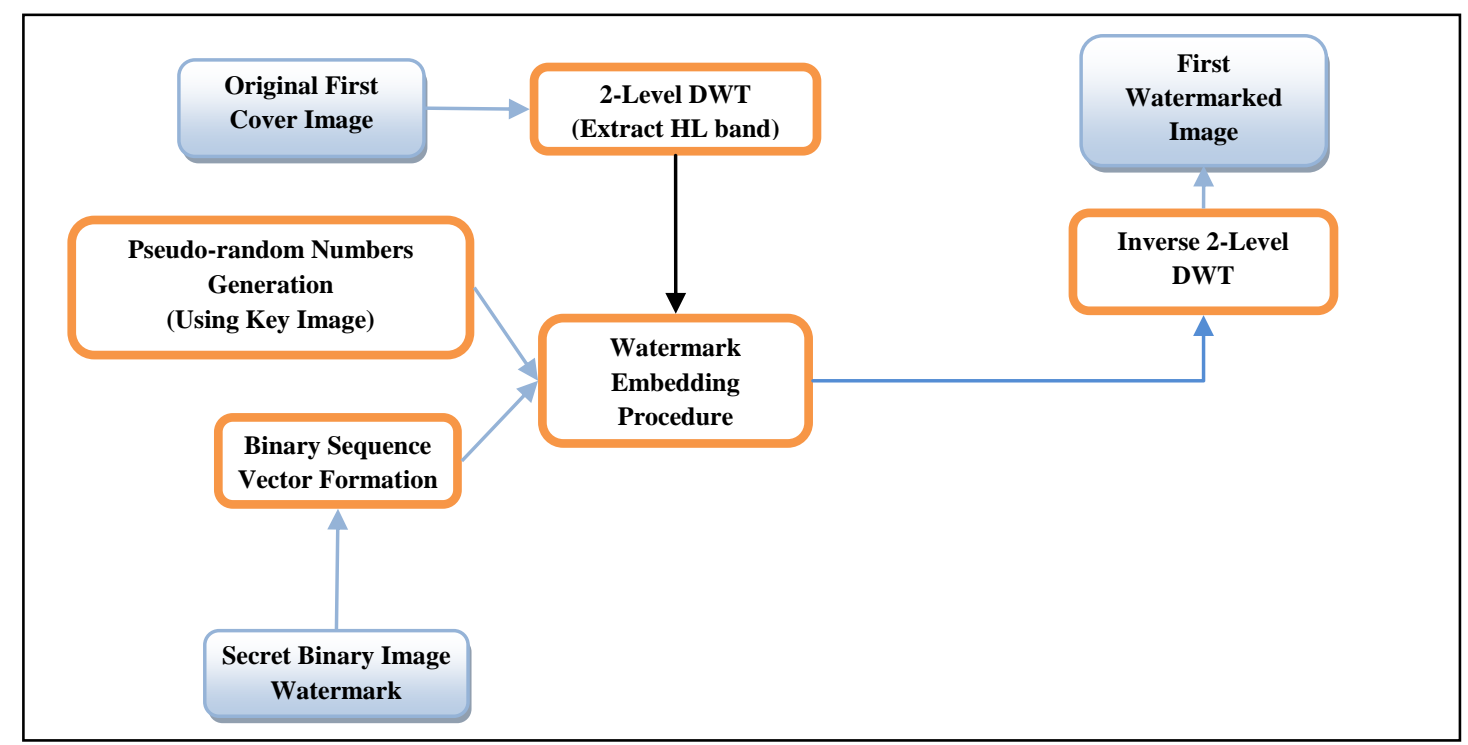

Fig.6. Stage-1 Watermarking Process (Blind)

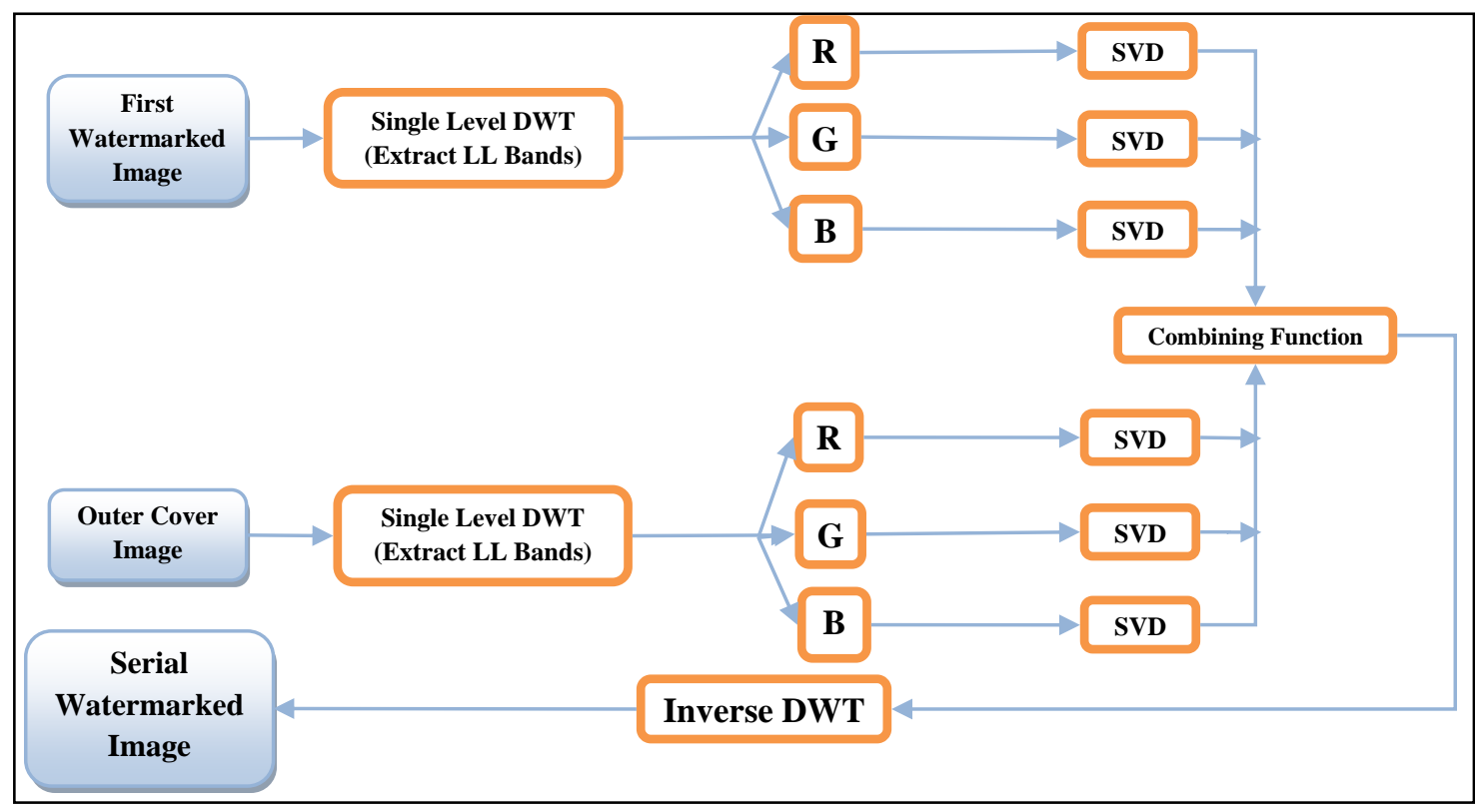

Fig.7. Stage-2 Watermarking Process (Non-Blind)

\section{B. Composite scheme for serial watermark extraction}

Secret binary image extraction from serial watermarked image is also done in two stages. Fig. 8 demonstrates watermark extraction process; Stage-1 of watermark extraction process uses non-blind watermark extraction technique. The first watermarked image is extracted from serial watermarked image using transformation tools DWT and SVD. Stage-2 uses blind watermark extraction technique with DWT tool; where the output of Stage- 1 is first secret watermarked image and is the input for Stage-2. The Output of Stage-2 will be an extracted secret binary image.
The Fig.9 depicts the Stage-1 of serial watermark extraction process. Here Separation function is for extracting first watermarked image from the serial watermarked image. To frame separation function, apply single level DWT on the copy of first watermarked image to get LL subband which contains most of the image information including hidden information. Then extract $\mathrm{R}$, $G$ and $B$ channels from LL subband. Apply SVD separately on each of R, G and B channel extracted from LL subband of copy of first watermarked image to get $S$ matrices on $\mathrm{S}^{\mathrm{R}}, \mathrm{S}^{\mathrm{G}}$, and $\mathrm{S}^{\mathrm{B}}$ channels separately and similarly get for $\mathrm{U}$ and $\mathrm{V}$ matrices also. Same procedure is applied on serial watermarked image also. Then 


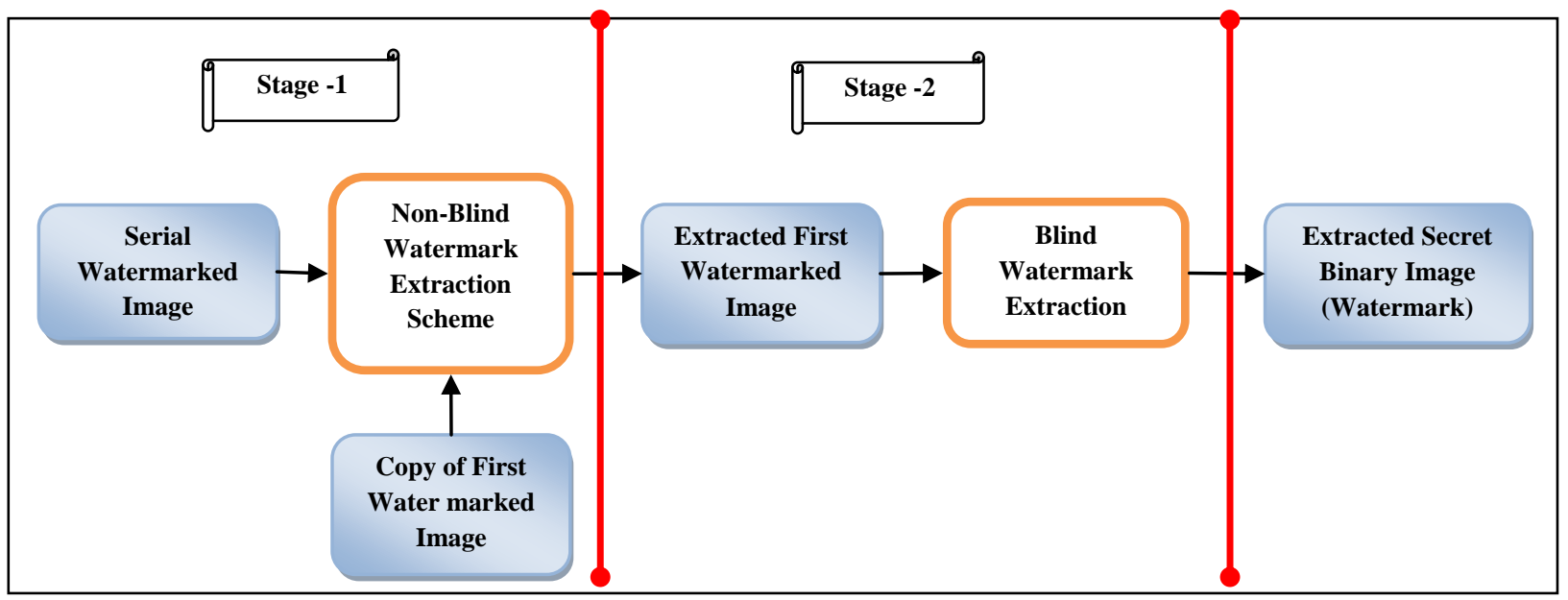

Fig.8. Composite scheme for serial watermark extraction

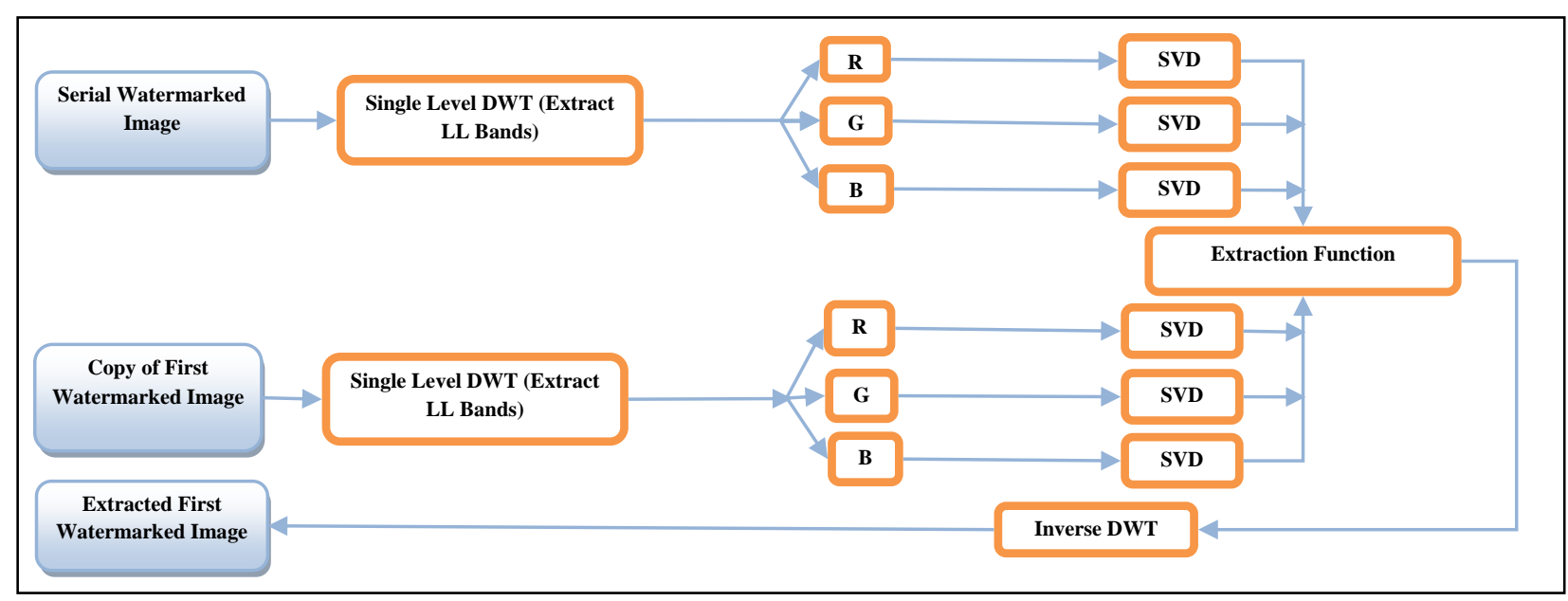

Fig.9. Stage-1 Watermark Extraction Process (Non-Blind)

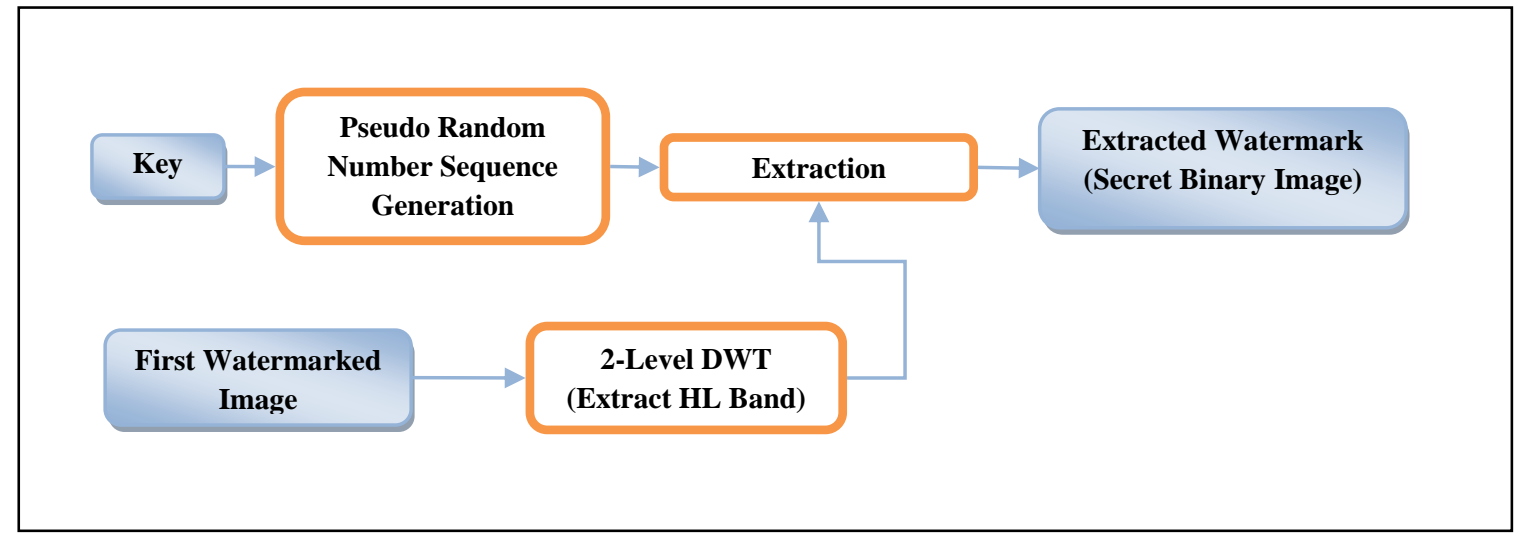

Fig.10. Stage-2 Watermark Extraction Process (Blind)

subtract copy of first watermarked image' $\left(S^{R}, S^{G}\right.$ and $\left.S^{B}\right)$ matrices from serial watermarked image' $\left(S^{R}, S^{G}\right.$ and $\left.S^{B}\right)$ matrices respectively to get embedded first watermarked image' $\left(S^{R}, S^{G}\right.$ and $\left.S^{B}\right)$. Multiply the respective $U, V$ matrices to get complete embedded first watermarked image (*Because of the nature of non-blind watermarking technique "copy of first watermarked image", "serial watermarked image" are taken here to get embedded "first watermarked image" from serial watermarked image). This whole process is presented in stage- 1 of Algorithm-2. Then apply 2-level DWT on extracted first watermarked image to locate HL2 sub-bands using Haar wavelets. Compute pseudo-random sequence generated with the help of key image used in the watermark embedding process. Scan the HL2 subband image using a pre-defined block of size $4 \times 4$ binary sequence coefficients. Calculate the correlation of the binary sequence block to pseudorandom sequence with extracted 
binary sequence coefficients and move to next block. This will lead to watermark extraction and is depicted in Fig. 10 and details in stage- 2 of Algorithm 2.

\section{Algorithms}

Abbreviations used in the algorithms

FCI - First Cover Image

SBI - Secret Binary Image

FWI - First Watermarked Image

SCI - Second Cover Image

SWI - Serial Watermarked Image

R, G, B - Red, Green, Blue channels of input color image

U, S, V - U - Left singular vectors, S - Diagonal singular vectors, V - Right singular vectors of SVD

Algorithm 1: Watermark Embedding

Input : i. First cover image (FCI).

ii. Secret binary image (SBI).

iii. Second cover image (SCI)

Output : Serial Watermarked image (SWI).

\section{Stage-1}

Start

Step.1: Decompose the FCI into four non-overlapping sub-bands using haar discrete wavelet transformation: $\mathrm{LL}_{1}^{\mathrm{FCI}}, \mathrm{HL} 1^{\mathrm{FCI}}, \mathrm{LH} 1^{\mathrm{FCI}}$, and $\mathrm{HH} 1^{\mathrm{FCI}}$

Step.2: Apply haar DWT again to sub-band $\mathrm{HL} 1^{\mathrm{FCI}}$ to get four smaller sub-bands and select the HL2 ${ }^{\mathrm{HL} \text { FCI }}$ sub-band.

Step.3: Divide the sub-band HL2 ${ }^{\text {HL FCI }}$ into predefined binary sequence structure of $4 \times 4$ blocks.

Step.4: Re-formulate the SBI image into a vector of zeros and ones.

Step.5: Generate two uncorrelated pseudorandom sequences of SBI. One sequence is used to embed the watermark bit 0 and the other sequence is sued to embed the watermark bit 1 .

Step.6: Embed the two pseudorandom sequences; sequence 0 and sequence 1 , with a gain factor some $\mathrm{k}, 4 \times 4$ blocks of the selected DWT subbands of the FCI.

If the secret watermark image bit is 0 then $\mathrm{X}^{\prime}=\mathrm{X}+\mathrm{k} \times$ pseudorandom sequence for 0 otherwise,

$\mathrm{X}^{\prime}=\mathrm{X}+\mathrm{k} \times$ pseudorandom sequence for 1

[ Where $\mathrm{X}$ ' represents the pseudorandom sequence embedded block. $X$ denotes the matrix of the coefficients of block. ]

Step.7: Apply the inverse DWT on the modified subband, to produce the FWI.

\section{Stage-2}

Step.8: Decompose the FWI into four sub-bands: FWI ${ }^{\mathrm{LL}}$, $\mathrm{FWI}^{\mathrm{HL}}, \mathrm{FWI}^{\mathrm{LH}}$, and $\mathrm{FWI}^{\mathrm{HH}}$ using Haar DWT.

Step.9: Get R, G, and B components from $\mathrm{FWI}^{\mathrm{LL}}$ as R $\left(\mathrm{FWI}^{\mathrm{LL}}\right), \mathrm{G}\left(\mathrm{FWI}^{\mathrm{LL}}\right)$ and $\mathrm{B}\left(\mathrm{FWI}^{\mathrm{LL}}\right)$

Step.10: Apply SVD to each of $\mathrm{R}\left(\mathrm{FWI}^{\mathrm{LL}}\right), \mathrm{G}\left(\mathrm{FWI}^{\mathrm{LL}}\right)$ and $\mathrm{B}\left(\mathrm{FWI}^{\mathrm{LL}}\right)$ of Step 9 . to get

$\mathrm{U}\left(\mathrm{R}\left(\mathrm{FWI}^{\mathrm{LL}}\right)\right), \mathrm{U}\left(\mathrm{G}\left(\mathrm{FWI}^{\mathrm{LL}}\right)\right)$ and $\mathrm{U}\left(\mathrm{B}\left(\mathrm{FWI}^{\mathrm{LL}}\right)\right)$

$\mathrm{S}\left(\mathrm{R}\left(\mathrm{FWI}^{\mathrm{LL}}\right)\right), \mathrm{S}\left(\mathrm{G}\left(\mathrm{FWI}^{\mathrm{LL}}\right)\right)$ and $\mathrm{S}\left(\mathrm{B}\left(\mathrm{FWI}^{\mathrm{LL}}\right)\right)$
$\mathrm{V}\left(\mathrm{R}\left(\mathrm{FWI}^{\mathrm{LL}}\right)\right), \mathrm{V}\left(\mathrm{G}\left(\mathrm{FWI}^{\mathrm{LL}}\right)\right)$ and $\mathrm{V}\left(\mathrm{B}\left(\mathrm{FWI}^{\mathrm{LL}}\right)\right)$

Step.11: Repeat Step. 8 to Step.10 for SCI also to get $\mathrm{U}\left(\mathrm{R}\left(\mathrm{SCI}^{\mathrm{LL}}\right)\right), \mathrm{U}\left(\mathrm{G}\left(\mathrm{SCI}^{\mathrm{LL}}\right)\right)$ and $\mathrm{U}\left(\mathrm{B}\left(\mathrm{SCI}^{\mathrm{LL}}\right)\right)$ $\mathrm{S}\left(\mathrm{R}\left(\mathrm{SCI}^{\mathrm{LL}}\right)\right), \mathrm{S}\left(\mathrm{G}\left(\mathrm{SCI}^{\mathrm{LL}}\right)\right)$ and $\mathrm{S}\left(\mathrm{B}\left(\mathrm{SCI}^{\mathrm{LL}}\right)\right)$ $\mathrm{V}\left(\mathrm{R}\left(\mathrm{SCI}^{\mathrm{LL}}\right)\right), \mathrm{V}\left(\mathrm{G}\left(\mathrm{SCI}^{\mathrm{LL}}\right)\right)$ and $\mathrm{V}\left(\mathrm{B}\left(\mathrm{SCI}^{\mathrm{LL}}\right)\right)$

Step.12: Now compute (Combining Function) $\mathrm{S} \_$new_R $=\left(\mathrm{S}\left(\mathrm{R}\left(\mathrm{SCI} \mathrm{I}^{\mathrm{LL}}\right)\right)\right)+\left(\mathrm{S}\left(\mathrm{R}\left(\mathrm{FWI}^{\mathrm{LL}}\right)\right) \times\right.$ gain_factor_2)

$\mathrm{S}$ nnew_G $=\left(\mathrm{S}\left(\mathrm{G}\left(\mathrm{SCI}{ }^{\mathrm{LL}}\right)\right)\right)+\left(\mathrm{S}\left(\mathrm{G}\left(\mathrm{FWI}^{\mathrm{LL}}\right)\right) \times\right.$ gain_factor_2)

$\mathrm{S} \_$new_B $=\left(\mathrm{S}\left(\mathrm{B}\left(\mathrm{SCI}^{\mathrm{LL}}\right)\right)\right)+\left(\mathrm{S}\left(\mathrm{B}\left(\mathrm{FWI}^{\mathrm{LL}}\right)\right) \times\right.$ gain_factor_2)

WI_R $=\mathrm{U}\left(\mathrm{R}\left(\mathrm{SCI}{ }^{\mathrm{LL}}\right)\right) \times \mathrm{S} \_$new_R $\times \mathrm{V}\left(\mathrm{R}\left(\mathrm{SCI}^{\mathrm{LL}}\right)\right)$ WI_G $=U\left(G\left(S C I^{\mathrm{LL}}\right)\right) \times$ S_new_G $\times V\left(G\left(S I^{\mathrm{LL}}\right)\right)$ WI_B $=\mathrm{U}\left(\mathrm{B}\left(\mathrm{SCI}^{\mathrm{LL}}\right)\right) \times \mathrm{S}$ new_B $\times \mathrm{V}(\mathrm{B}$ $\left.\left(\mathrm{SCI}^{\mathrm{LL}}\right)\right)$

newhost_LL= Concatnate (WI_R, WI_G, WI_B)

Step.13: Apply inverse Haar DWT on newhost_LL to get SWI

Stop.

The Algorithm 1 uses blind watermarking technique in Stage-1 to embed secret binary image into first cover image and Non-blind watermarking technique in Stage-2 to embed first watermarked image into second cover image.

Algorithm 2: Watermark extraction Input: i. Serial Watermarked image ii. Copy of First watermarked image Output: Extracted secret binary image Stage-1

Start

Step.1: Decompose the FWI into four sub-bands: FWI ${ }^{\mathrm{LL}}$, $\mathrm{FWI}^{\mathrm{HL}}, \mathrm{FWI}^{\mathrm{LH}}$, and $\mathrm{FWI}^{\mathrm{HH}}$ using Haar DWT.

Step.2: Get R, G, and B components from $\mathrm{FWI}^{\mathrm{LL}}$ as R $\left(\mathrm{FWI}^{\mathrm{LL}}\right), \mathrm{G}\left(\mathrm{FWI}^{\mathrm{LL}}\right)$ and $\mathrm{B}\left(\mathrm{FWI}^{\mathrm{LL}}\right)$

Step.3: Apply SVD to each of R (FWI $\left.{ }^{\mathrm{LL}}\right), \mathrm{G}\left(\mathrm{FWI}^{\mathrm{LL}}\right)$ and $\mathrm{B}\left(\mathrm{FWI}^{\mathrm{LL}}\right)$ of Step 9 . to get $\mathrm{U}\left(\mathrm{R}\left(\mathrm{FWI}^{\mathrm{LL}}\right)\right), \mathrm{U}\left(\mathrm{G}\left(\mathrm{FWI}^{\mathrm{LL}}\right)\right)$ and $\mathrm{U}\left(\mathrm{B}\left(\mathrm{FWI}^{\mathrm{LL}}\right)\right)$ $\mathrm{S}\left(\mathrm{R}\left(\mathrm{FWI}^{\mathrm{LL}}\right)\right), \mathrm{S}\left(\mathrm{G}\left(\mathrm{FWI}^{\mathrm{LL}}\right)\right)$ and $\mathrm{S}\left(\mathrm{B}\left(\mathrm{FWI}^{\mathrm{LL}}\right)\right)$ $\mathrm{V}\left(\mathrm{R}\left(\mathrm{FWI}^{\mathrm{LL}}\right)\right), \mathrm{V}\left(\mathrm{G}\left(\mathrm{FWI}^{\mathrm{LL}}\right)\right)$ and $\mathrm{V}\left(\mathrm{B}\left(\mathrm{FWI}^{\mathrm{LL}}\right)\right)$

Step.4: Repeat Step.1 to Step.3 for SWI also to get $\mathrm{U}\left(\mathrm{R}\left(\mathrm{SWI} \mathrm{IL}^{\mathrm{LL}}\right)\right), \mathrm{U}\left(\mathrm{G}\left(\mathrm{SWI} \mathrm{I}^{\mathrm{LL}}\right)\right)$ and $\mathrm{U}\left(\mathrm{B}\left(\mathrm{SWI}^{\mathrm{LL}}\right)\right)$ $\mathrm{S}\left(\mathrm{R}\left(\mathrm{SWI}^{\mathrm{LL}}\right)\right), \mathrm{S}\left(\mathrm{G}\left(\mathrm{SWI} \mathrm{I}^{\mathrm{LL}}\right)\right)$ and $\mathrm{S}\left(\mathrm{B}\left(\mathrm{SWI}^{\mathrm{LL}}\right)\right)$ $\mathrm{V}\left(\mathrm{R}\left(\mathrm{SWI}^{\mathrm{LL}}\right)\right), \mathrm{V}\left(\mathrm{G}\left(\mathrm{SWI}^{\mathrm{LL}}\right)\right)$ and $\mathrm{V}\left(\mathrm{B}\left(\mathrm{SWI}^{\mathrm{LL}}\right)\right)$

Step.5: Now compute (Extraction Function) S_new_R $=\left(\mathrm{S}\left(\mathrm{R}\left(\mathrm{SWI}^{\mathrm{LL}}\right)\right)-\mathrm{S}\left(\mathrm{R}\left(\mathrm{FWI}^{\mathrm{LL}}\right)\right)\right)$ /Const

S_new_G $=\left(\mathrm{S}\left(\mathrm{G}\left(\mathrm{SWI}^{\mathrm{LL}}\right)\right)-\mathrm{S}\left(\mathrm{G}\left(\mathrm{FWI}^{\mathrm{LL}}\right)\right)\right)$ /Const

S_new_B $=\left(\mathrm{S}\left(\mathrm{B}\left(\mathrm{SWI}{ }^{\mathrm{LL}}\right)\right)-\mathrm{S}\left(\mathrm{B}\left(\mathrm{FWI}^{\mathrm{LL}}\right)\right)\right) /$ Const

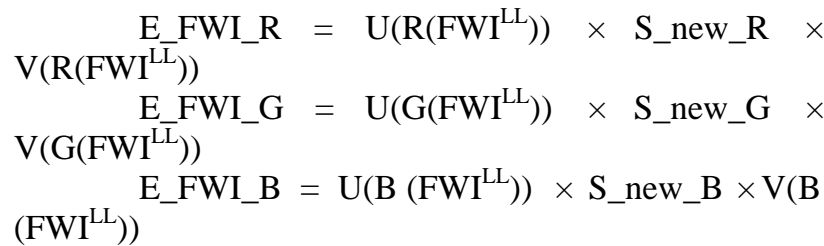


newhost_LL $=$ Concatenate (E_FWI_R, E_FWI_G, E_FWI_B)

Step.6: Apply inverse Haar DWT on newhost_LL to get E_FWI

\section{Stage-2}

Step.7: Decompose the E_FWI of step 6 into four nonoverlapping multi-resolution

subbands: $\mathrm{LL1}^{\mathrm{E} \_\mathrm{FWI}}, \mathrm{HL1}^{\mathrm{E}-\mathrm{FWI}}, \mathrm{LH}^{\mathrm{E} \_\mathrm{FWI}}$, and $\mathrm{HH} 1^{\mathrm{E} \_ \text {FWI }}$ using DWT.

Step.8: Apply DWT to HL1 ${ }^{\text {E_FWI }}$ to get four smaller subbands, and choose the sub-band $\mathrm{HL}_{2}{ }^{\mathrm{E}-\mathrm{FWI}}$

Step.9: Divide the sub-band HL2 ${ }^{\mathrm{E}-\mathrm{FWI}}$ into predefined binary sequence structure of $4 \times 4$ blocks.

Step.10: Regenerate the two pseudorandom sequences for 0 and 1 using the same seed used in the watermark embedding procedure of Stage- 1 .

Step.11: For each block in the sub-band HL2 $2^{\text {E_FWI }}$, calculate the correlation between the elements coefficients and the two generated pseudorandom sequences for 0 and 1 .

If the correlation with the pseudorandom sequence 0 was higher than the correlation with pseudorandom sequence 1 , then the extracted watermark bit is considered 0

Otherwise the extracted watermark is considered

1.

Step.12: Reconstruct the watermark using the extracted watermark bits, and compute the similarity between the original and extracted watermarks.

Stop.

The Algorithm 2 uses the Non-blind watermarking technique in Stage-1to extract first watermarked image from the second image and Blind watermarking technique in Stage-2 to extract the secret image from first watermarked image. The abbreviations used in the algorithm are mentioned in the beginning of the Algorithm part.

\section{RESUltS AND DisCUSSIONS}

The scheme proposed here is a robust digital image watermarking scheme which can be more suitable for various applications. The proposed scheme is nonreversible digital image watermarking which cannot restore the original image but it restores original watermark (secret binary image) in a lossless manner for no attacks but it also recovers the original secret binary image (watermark) in a case of various attacks. Moreover, the proposed method uses both blind and non-blind techniques to embed the watermark into a cover image. The blind watermarking technique proposed here needs a key to embed a secret binary image into the first cover image to get the first watermarked image. The first watermarked image is embedded into the second cover image to get serial watermarked image. The performance of proposed scheme has been evaluated in terms of quality of the watermarked image by computing peak signal to noise ratio (PSNR) and robustness of the watermarked image by measuring correlation. Table. 1 shows the images considered here for testing the proposed scheme. The color images of size $512 \times 512$ as a first cover image, second cover image and the binary image of size $50 \times 20$ is considered as a secret binary image (watermark). For copyright protection, security of the secret image is an important factor.

Table.1 Inputs for the proposed technique

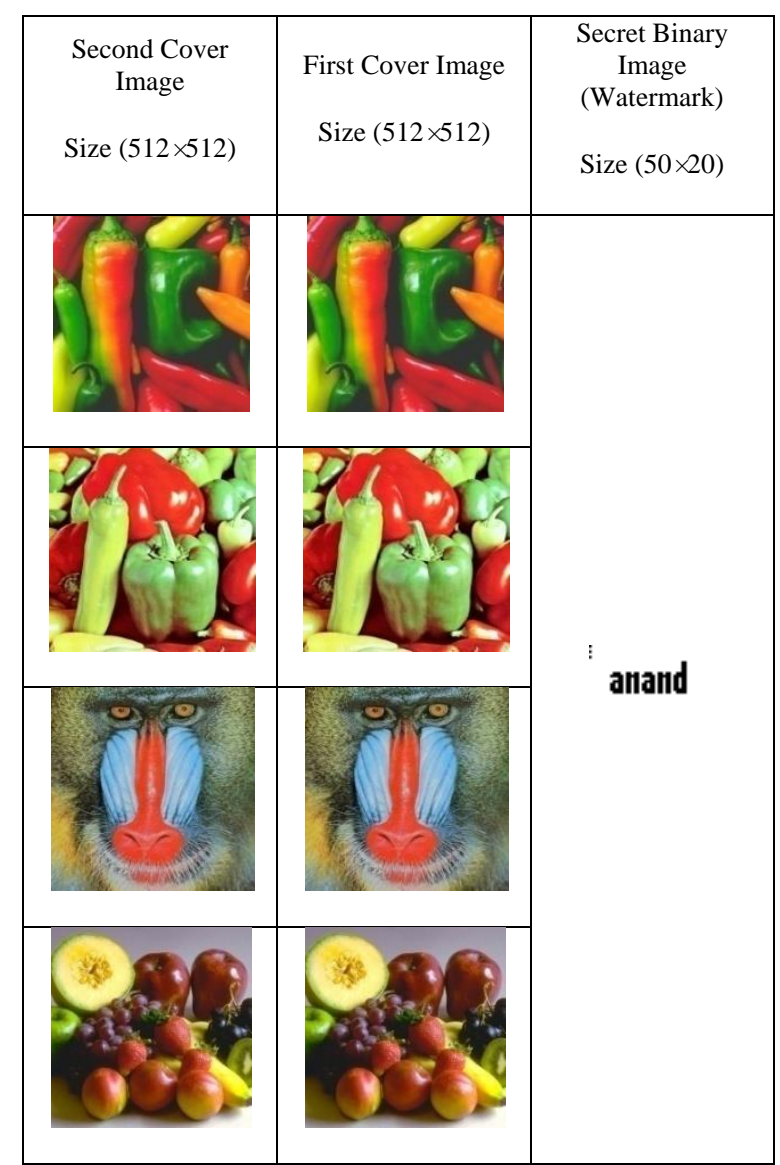

\section{Analysis of security}

One of the important indicators of watermarking system is security; if it is insecure then definitely it loses its value for practical applications. The proposed scheme achieves the security parameter effectively, including the position of embedding a secret binary image into first cover image and procedure to embed. The stage- 1 of watermark embedding procedure uses HL sub-bands of the first cover image by DWT to hide the binary image (secret binary image vector of 0 and 1) using key and $4 \times 4$ binary sequence block mapped on to HL sub-band image of the first cover image. The stage- 2 of watermark embedding procedure takes the first watermarked image, second cover image and extracts LL sub-bands by DWT. The R, G and B channels are extracted from LL subbands of both first watermarked image and second cover image. The SVD [12] is applied to R, G and B of each of first watermarked image, second cover image and combining the diagonal singular values of each image to get serial watermarked image. Similarly while extracting the secret binary image we need a copy of first watermarked image and serial watermarked image are 
needed to get a first watermarked image which contains a secret binary image. Then by using a secret key, the secret binary image can be extracted as it is. The major disadvantage of using SVD and in connection with nonblind technique is the production of false positive/negative error [18]. The root cause of the falsepositive/negative problem is that the SVD vectors $U$ and $\mathrm{V}$ influence the watermark being extracted from the watermarked image [12] [18][20][21]. This cause will extract the secret binary image as it is from serial watermarked image in spite of various noise attacks.

\section{Analysis of Fidelity (Imperceptibility)}

The perceptual similarity between the second cover image and serial watermarked image can be verified by measuring Peak Signal-to-Noise Ratio (PSNR) (Eq. 6) in decibels $(\mathrm{dB})$. Table. 2 and Table. 3 shows the results of serial watermarking scheme and advantages of combining blind and non-blind techniques. Table. 2 and Table. 3 also shows the extracted secret binary image (watermark), a correlation between original watermark and extracted watermark. When the same type of first cover image and second cover image are used for serial digital image watermarking, we got the perceptual similarity as PSNR $=$ Infinity $\mathrm{dB}$. The effect of different first cover image and second cover image on perceptual similarity is also noted in Table. 2 and Table.3. The image here with 8 bits per pixel contains integers from 0 to 255 (B) PSNR will be given as eq. 6 .

$$
\text { PSNR }=20 \log _{10} \frac{\left(2^{\mathrm{B}}-1\right)}{\sqrt{\mathrm{MSE}}}
$$

The mean square error (MSE) is the squared norm of the difference between the data $\mathrm{x}$ and the approximation $\hat{\mathrm{x}}$ divided by the number of elements.

Where

$$
\text { MSE }=\frac{\|x-\hat{x}\|^{2}}{N}
$$

To compare the correlation between original secret binary image (watermark) and extracted secret binary image (watermark) we use the formula as depicted in eq.7.

$$
r=\frac{\sum_{i}\left(x_{i}-x_{m}\right)\left(y_{i}-y_{m}\right)}{\sqrt{\sum_{i}\left(x_{i}-x_{m}\right)^{2}} \sqrt{\sum_{i}\left(y_{i}-y_{m}\right)^{2}}}
$$

Where $x_{i}$ and $y_{i}$ are intensity values of $i^{\text {th }}$ pixel of an original secret binary image and extracted secret binary image, $x_{m}$ and $y_{m}$ are the mean intensity values of an original secret binary image and the extracted secret binary image.

The results shown in Table. 2 and Table. 3 are presented by varying the gain factor in both stage- 1 and stage- 2 of the proposed scheme. When we increase the gain factor in stage- 1 of watermarking process, it provides more strength (intensity is increased) to the hidden binary image leading to security. When we decrease the gain factor in stage-2, it leads to the decrease of the intensity value of first watermarked image; so when we embed first watermarked image into a second cover image, the perceptual similarity between serial watermarked image

\begin{tabular}{|c|c|c|c|c|c|c|}
\hline $\begin{array}{l}\text { First } \\
\text { Cover } \\
\text { Image }\end{array}$ & $\begin{array}{l}\text { Secret } \\
\text { Binary } \\
\text { Image }\end{array}$ & $\begin{array}{c}\text { First } \\
\text { Watermarke } \\
\text { d Images }\end{array}$ & $\begin{array}{l}\text { Second } \\
\text { Cover } \\
\text { Image }\end{array}$ & $\begin{array}{c}\text { Serial } \\
\text { Watermarked } \\
\text { Image }\end{array}$ & $\begin{array}{c}\text { Perceptual Similarity Between } \\
\text { Second Cover Image and Serial } \\
\text { Watermarked Image PSNR in dB } \\
\text { (Proposed Scheme) }\end{array}$ & $\begin{array}{l}\text { Correlation Between } \\
\text { Secret Watermark and } \\
\text { Extracted Secret } \\
\text { Watermark } \\
\text { (Proposed Scheme) } \\
\end{array}$ \\
\hline \multicolumn{3}{|c|}{ Stage- 1 Gain factor $=10$} & & & \multicolumn{2}{|c|}{ Stage- 2 Gain factor $=1 / 10$} \\
\hline & \multirow{4}{*}{ anand } & & & & 25.6160 & 0.6635 \\
\hline & & & & & 26.4218 & 0.5040 \\
\hline & & & & & 26.9022 & 0.8157 \\
\hline & & & & & 28.0664 & 0.9296 \\
\hline \multicolumn{5}{|c|}{ Stage -1 Gain factor $=50$} & \multicolumn{2}{|c|}{ Stage- 2 Gain factor $=1 / 10$} \\
\hline & anand & & & & 25.6176 & 0.9931 \\
\hline
\end{tabular}
and second cover image increases.

Table.2 The results of Composite Serial Digital Image Watermarking (Varying Stage-1 Gain factor and keeping Stage-2 Gain factor constant) 


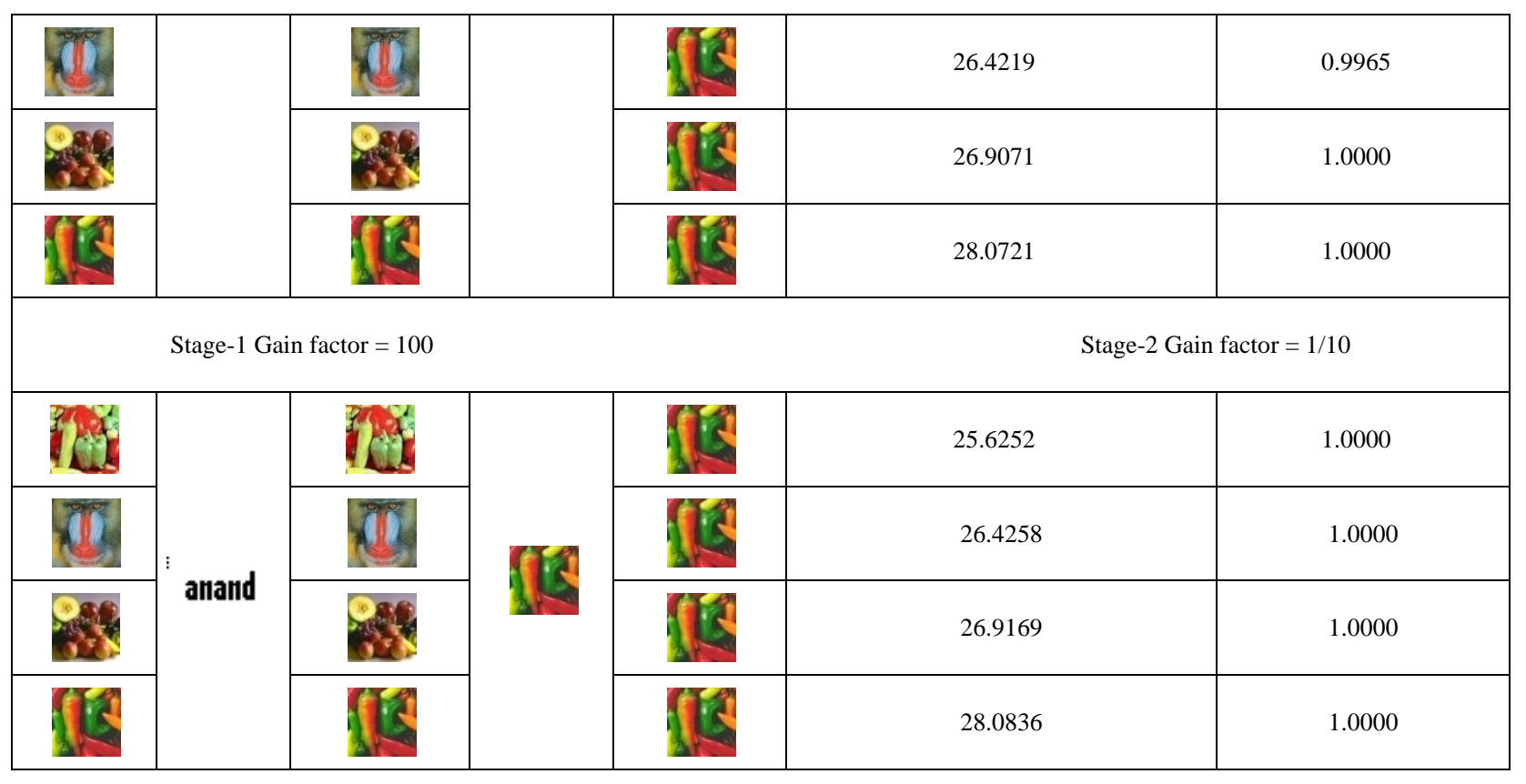

Table 3. The results of Composite Serial Digital Image Watermarking (Varying Stage-2 Gain factor and keeping Stage-1 Gain factor constant)

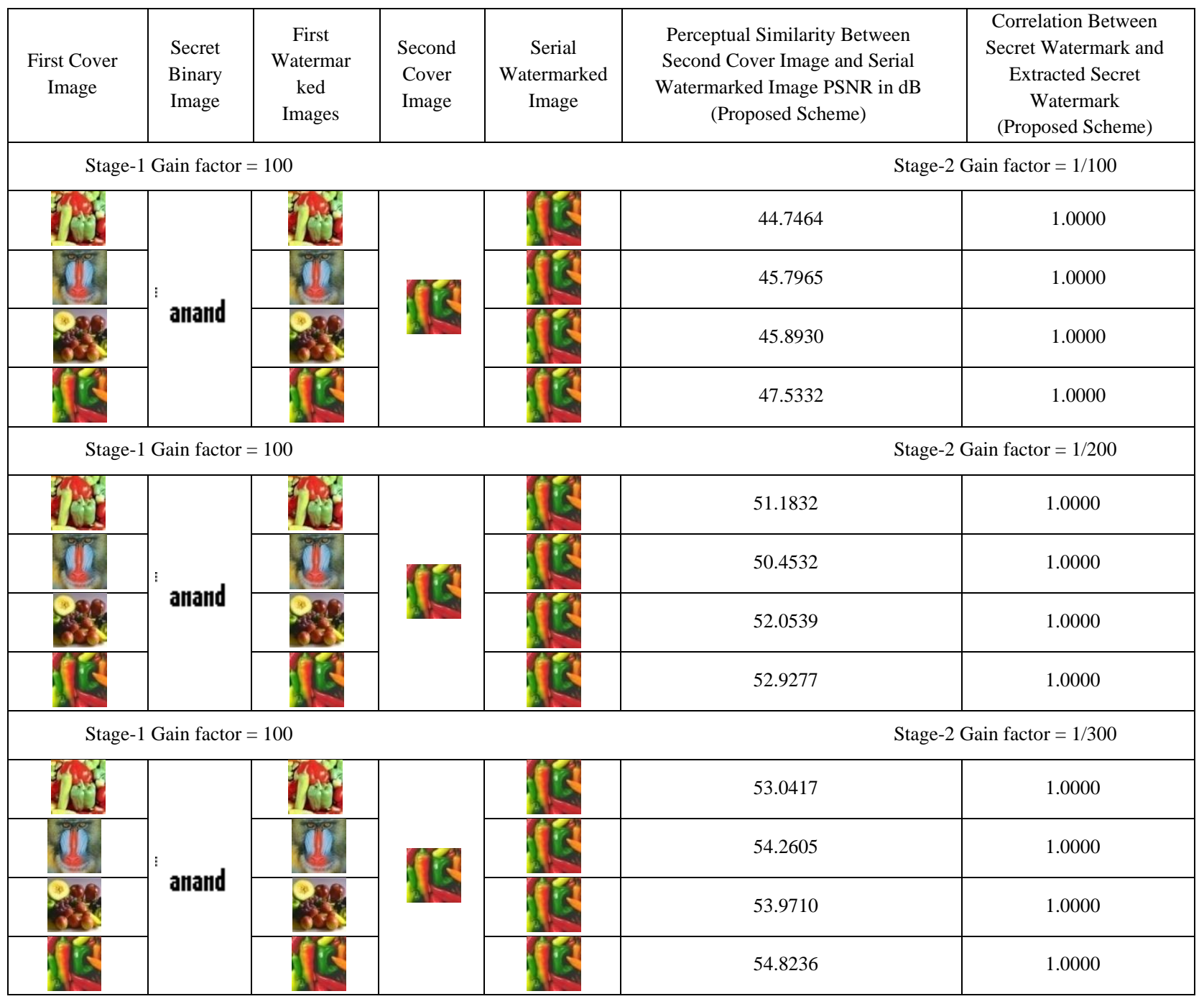




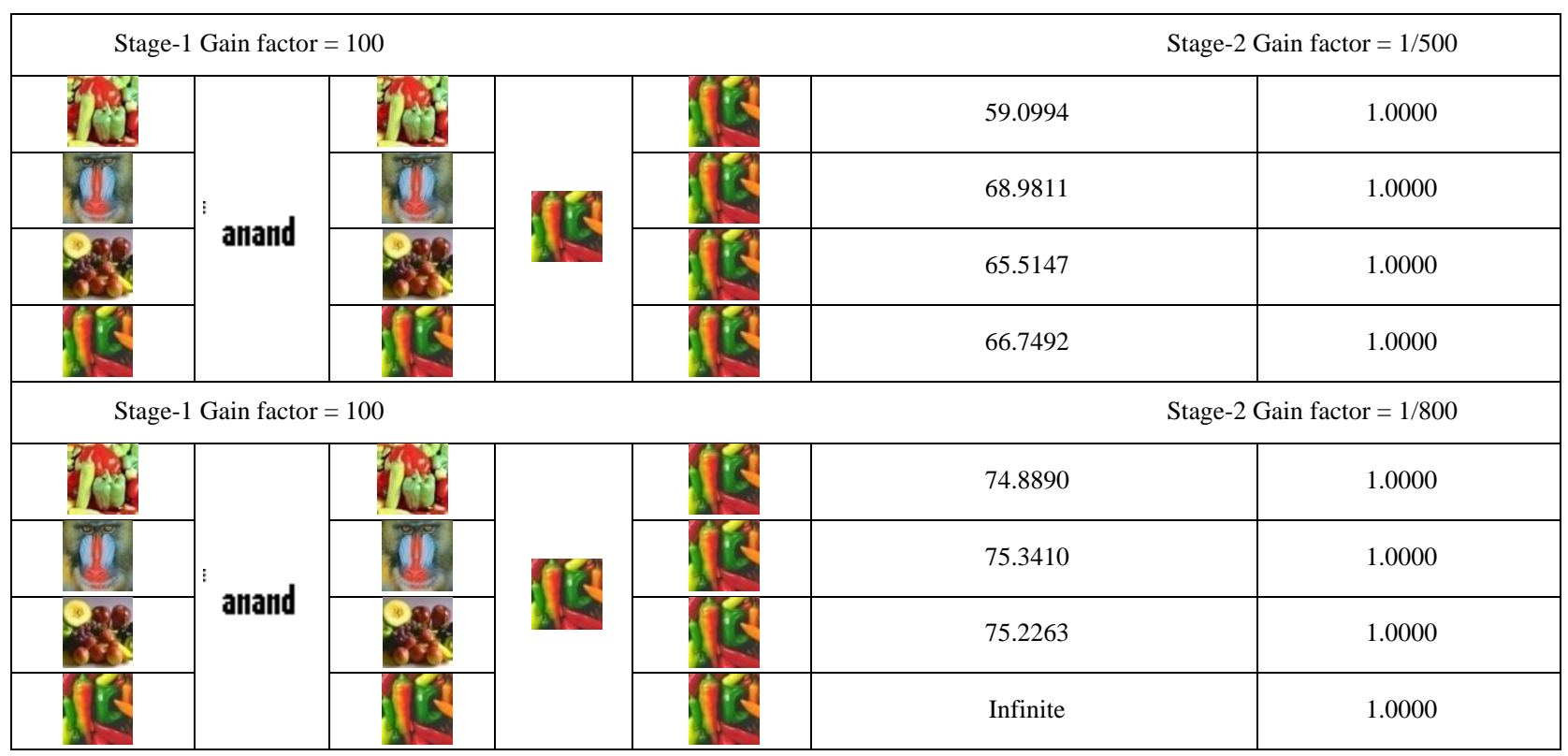

Table.4 The comparison between perceptual similarity results of proposed scheme and various schemes of reference on different set of cover images and watermarks

\begin{tabular}{|c|c|c|c|c|c|}
\hline $\begin{array}{l}\text { Perceptual Similarity } \\
\text { Between Second } \\
\text { Cover Image and } \\
\text { Serial Watermarked } \\
\text { Image } \\
\text { (PSNR in dB) } \\
\text { (Proposed Scheme) }\end{array}$ & $\begin{array}{l}\text { Perceptual Similarity } \\
\text { Between Cover Image } \\
\text { and Watermarked } \\
\text { Image } \\
\text { (PSNR in dB) } \\
\text { Scheme of Reference } \\
2\end{array}$ & $\begin{array}{c}\text { Perceptual Similarity } \\
\text { Between Cover Image } \\
\text { and Watermarked } \\
\text { Image } \\
\text { (PSNR in dB) } \\
\text { Scheme of Reference } \\
3\end{array}$ & $\begin{array}{c}\text { Perceptual } \\
\text { Similarity } \\
\text { Between Cover } \\
\text { Image and } \\
\text { Watermarked } \\
\text { Image } \\
\text { (PSNR in dB) } \\
\text { Scheme of } \\
\text { Reference } 4\end{array}$ & $\begin{array}{c}\text { Perceptual } \\
\text { Similarity } \\
\text { Between Cover } \\
\text { Image and } \\
\text { Watermarked } \\
\text { Image } \\
\text { (PSNR in dB) } \\
\text { Scheme of } \\
\text { Reference } 5\end{array}$ & $\begin{array}{c}\text { Perceptual } \\
\text { Similarity } \\
\text { Between Cover } \\
\text { Image and } \\
\text { Watermarked } \\
\text { Image } \\
\text { (PSNR in dB) } \\
\text { Scheme of } \\
\text { Reference } 6\end{array}$ \\
\hline 25.6160 & 42.5213 & 49.8664 & & 26.16 & 48.16 \\
\hline To & To & To & 58.91 & To & To \\
\hline 75.2263 & 48.3773 & 50.0839 & & 57.01 & 48.99 \\
\hline
\end{tabular}

Table.5 The comparison between proposed watermark extraction scheme and various schemes of reference on different set of cover images and watermarks

\begin{tabular}{|c|c|c|c|}
\hline $\begin{array}{c}\text { Correlation Between } \\
\text { Secret Binary Image } \\
\text { (Watermark) and } \\
\begin{array}{c}\text { Extracted Secret Binary } \\
\text { Image (Watermark) } \\
\text { (Proposed Scheme) }\end{array}\end{array}$ & $\begin{array}{c}\text { Correlation Between } \\
\text { Watermark and Extracted } \\
\text { Watermark } \\
\text { (Scheme of Reference 3) }\end{array}$ & $\begin{array}{c}\text { Correlation Between } \\
\text { Watermark and Extracted } \\
\text { Watermark } \\
\text { (Scheme of Reference 4) }\end{array}$ & $\begin{array}{c}\text { Correlation Between } \\
\text { Watermark and Extracted } \\
\text { Watermark } \\
\text { (Scheme of Reference 5) }\end{array}$ \\
$\begin{array}{c}\text { Extracted Watermark } \\
\text { (Scheme of Reference 6) }\end{array}$ \\
\hline 1.0000 & 1.0000 & 1.0000 & 1.0000 \\
\hline
\end{tabular}

Table.4 shows the range of PSNR values on various types of images with other schemes presented in reference and Table.5 shows the range of correlation between various types of watermarks and extracted watermarks with other schemes presented in the reference. The proposed scheme uses two stage watermarking scheme that is the content embedded in serial watermarked image is the combination of secret binary image and first cover image. Hence from Table.3, it can be observed that the PSNR values of proposed scheme are lesser than the range from other schemes.

Capacity Analysis
In the case of blind watermarking technique of stage-1, the original first cover image is of size $512 \times 512$, after applying 2-level DWT we get the image of size $128 \times 128$, then by $4 \times 4$ block processing technique giving the total 1024 blocks. Hence here the small secret binary image of size $50 \times 20$ is considered. However non-blind watermarking technique of stage- 2 uses two images (first watermarked image and second cover image) of the same size and one level DWT, SVD on both of the input images.

Robustness Analysis 
Stage-1 watermarking embeds secret binary image into first cover image to get a first watermarked image, the visual quality is least bothered here. But whereas stage-2 concentrates on visual quality and this can be achieved by embedding first watermarked image into a second cover image using DWT and SVD. Table.6 and Table.7 shows the effect of various attacks on the secret binary image retrieval process and Table. 8 shows the comparison between proposed scheme and the scheme of referenced articles on various noises.

Table.6 The result of secret binary image extraction process on various attacks

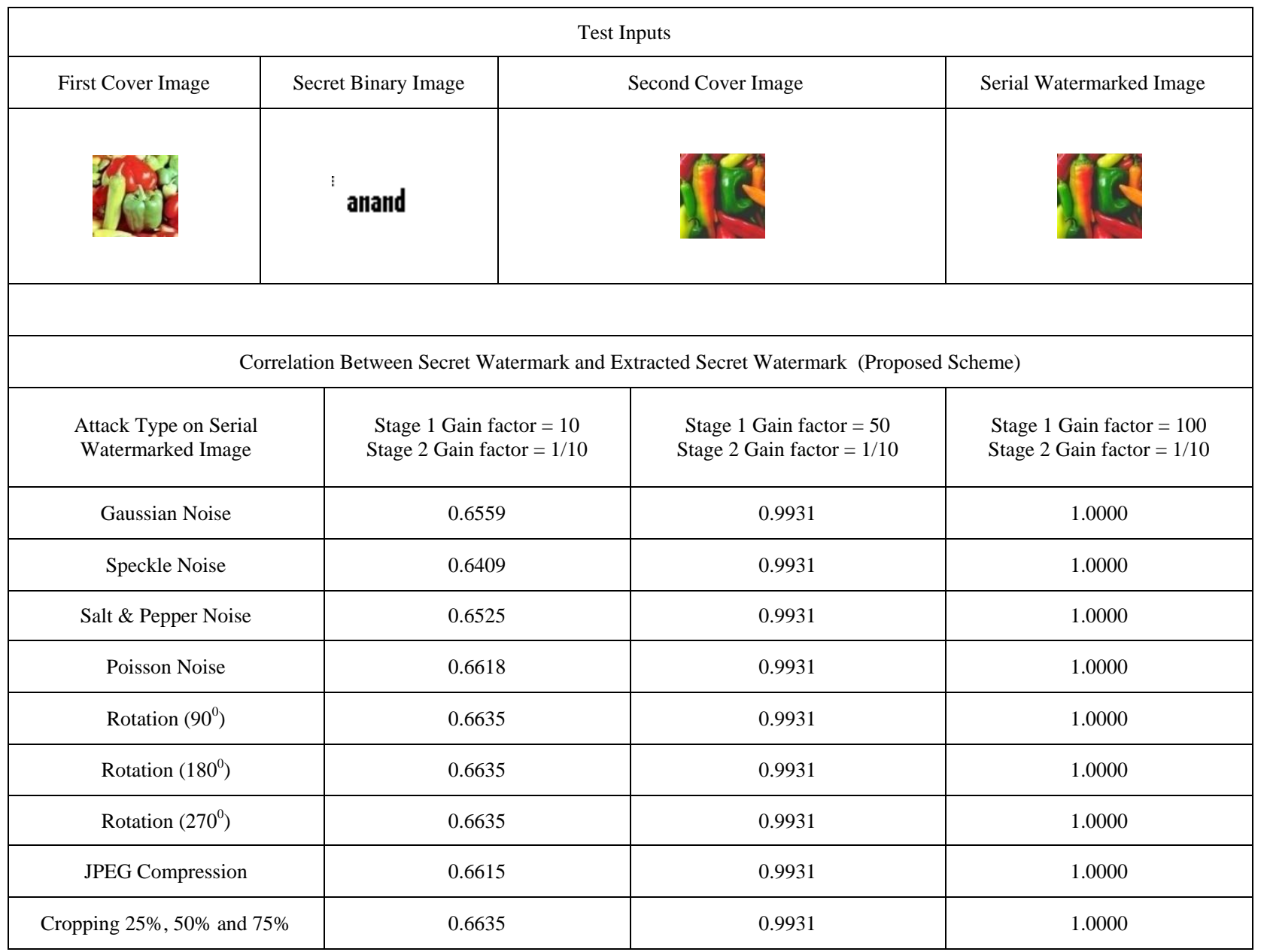

\section{CONCLUSION}

The proposed watermark embedding and watermark extraction procedure uses DWT and SVD transformations. The procedure depicted here embeds a secret binary image into a first cover image to form first watermarked image. The first watermarked image is again embedded into a second cover image to get serial watermarked image. We got the PSNR value as Infinite $\mathrm{dB}$ in the case of same types of first and second cover images. And the extracted secret watermark correlation as 1.0000. This scheme is robust against attacks like JPEG compression, rotation, cropping and addition of Gaussian, Poisson, salt \& pepper, and speckle noises. In all the mentioned attacks, the secret watermark is extracted as it is, giving correlation as 1.0000. The different first cover image and second cover image yields PSNR values ranging from 25.6160 to $75.2263 \mathrm{db}$ and extracted secret watermark correlation nearly measured as 1.0000. The usage of gain factor in Stage-1 and Stage2 has an impact on the results obtained from this approach. The increase of gain factor in Stage-1will improves the robustness drastically and a significant decrease of gain factor in Stage-2 will increase perceptual similarity between serial watermarked image and second cover image. The usage of SVD in stage- 2 of the algorithm greatly influences the false positive/ negative results. 
Table.7 The result of secret binary image extraction process on various attacks

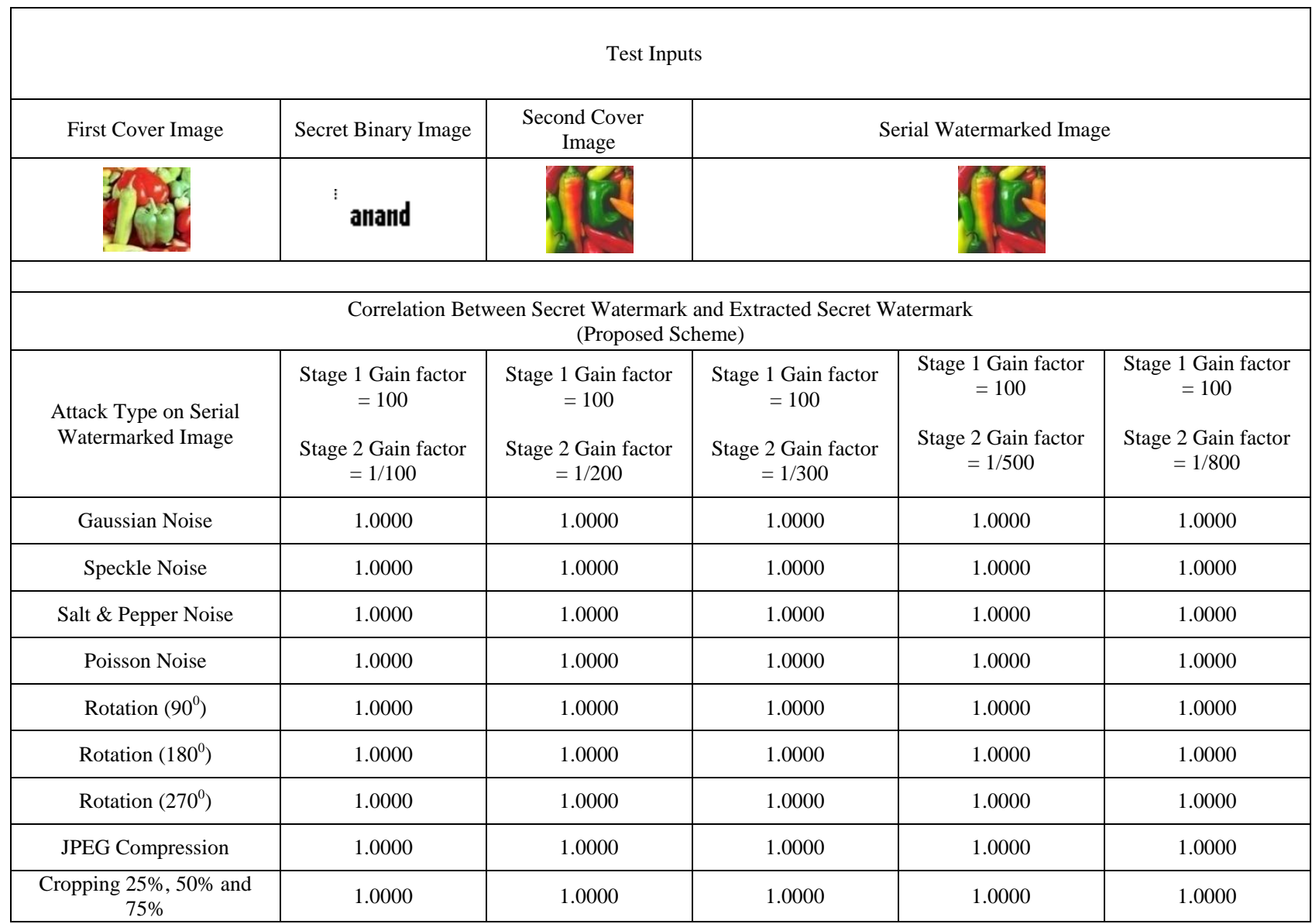

Table. 8 The comparison between proposed watermark extraction scheme and various schemes of reference on various types of attacks (Gaussian, Speckle, Poisson, Salt \& Pepper, Rotation and JPEG compression etc)

\begin{tabular}{|c|c|c|c|c|}
\hline \multicolumn{5}{|c|}{$\begin{array}{l}\text { The range of Correlation Between Watermark and Extracted Watermark } \\
\text { (On different set of color cover images and watermarks) }\end{array}$} \\
\hline Proposed Scheme & Scheme of Reference 3 & Scheme of Reference 4 & Scheme of Reference 5 & Scheme of Reference 6 \\
\hline 1.0000 to 1.0000 & $\begin{array}{c}0.86 \\
\text { to } \\
1.0000\end{array}$ & $\begin{array}{c}0.56 \\
\text { to } \\
1.0000\end{array}$ & $\begin{array}{c}0.487 \\
\text { to } \\
1.0000\end{array}$ & $\begin{array}{c}0.760 \\
\text { to } \\
0.997\end{array}$ \\
\hline
\end{tabular}

\section{REFERENCES}

[1] Liu, X., Han, G., Wu, J., Shao, Z., Coatrieux, G., \& Shu, H. (2017). Fractional Krawtchouk transform with an application to image watermarking. IEEE Transactions on Signal Processing.

[2] Hua, K. L., Dai, B. R., Srinivasan, K., Hsu, Y. H., \& Sharma, V. (2017). A hybrid NSCT domain image watermarking scheme. EURASIP Journal on Image and Video Processing, 2017(1), 10.

[3] Su, Q., \& Chen, B. (2017). Robust color image watermarking technique in the spatial domain. Soft Computing, 1-16.

[4] Kandi, H., Mishra, D., \& Gorthi, S. R. S. (2017). Exploring the learning capabilities of convolutional neural networks for robust image watermarking. Computers \& Security, 65, 247-268.

[5] Abdelhakim, A. M., Saleh, H. I., \& Nassar, A. M. (2017). A quality guaranteed robust image watermarking optimization with Artificial Bee Colony. Expert Systems with Applications, 72, 317-326.

[6] Wang, C., Wang, X., Zhang, C., \& Xia, Z. (2017). Geometric correction based color image watermarking using fuzzy least squares support vector machine and Bessel K form distribution. Signal Processing, 134, 197208.

[7] Etemad, E., Samavi, S., Soroushmehr, S. R., Karimi, N., Etemad, M., Shirani, S., \& Najarian, K. (2017). Robust image watermarking scheme using bit-plane of hadamard coefficients. Multimedia Tools and Applications, 1-23.

[8] Qin, C., Ji, P., Wang, J., \& Chang, C. C. (2017). Fragile image watermarking scheme based on VQ index sharing and self-embedding. Multimedia Tools and Applications, 76(2), 2267-2287.

[9] Zhang, Z., Wu, L., Yan, Y., Xiao, S., \& Sun, H. (2017). An improved reversible image watermarking algorithm based on difference expansion. International Journal of Distributed Sensor Networks, 13(1), 1550147716686577. 
[10] Hierarchical Recovery for Tampered Images Based on Watermark Self-Embedding. Displays.

[11] Qin, C., Wang, H., Zhang, X., \& Sun, X. (2016). Selfembedding fragile watermarking based on reference-data interleaving and adaptive selection of embedding mode. Information Sciences, 373, 233-250.

[12] Ansari, I. A., \& Pant, M. (2016). Multipurpose image watermarking in the domain of DWT based on SVD and ABC. Pattern Recognition Letters.

[13] Sheth, R. K., \& Nath, V. V. (2016, April). Secured digital image watermarking with discrete cosine transform and discrete wavelet transform method. In Advances in Computing, Communication, \& Automation (ICACCA)(Spring), International Conference on (pp. 1-5). IEEE.

[14] Ali Al-Haj (2007) Combined DWT-DCT Digital Image Watermarking. Journal of Computer Science, 3(9), 740746, ISSN: 1549-3636.

[15] http://in.mathworks.com/matlabcentral/fileexchange/4505 2-color-image-dwt-svd-watermarking.

[16] Xia, X. G., Boncelet, C. G., \& Arce, G. R. (1998). Wavelet transform based watermark for digital images. Optics Express, 3(12), 497-511.

[17] Cox, I., Miller, M., Bloom, J., Fridrich, J., \& Kalker, T. (2007). Digital watermarking and steganography. Morgan Kaufmann.

[18] Zear, A., Singh, A. K., \& Kumar, P. (2016). A proposed secure multiple watermarking technique based on DWT, DCT and SVD for application in medicine. Multimedia Tools and Applications, 1-20.

[19] Anuja Dixit \& Rahul Dixit. (2017). "A Review on Digital Image Watermarking Techniques." International Journal of Image, Graphics and Signal Processing (MECS) 9, no. 4: 56-66.

[20] Subin Bajracharya \& Roshan Koju. "An Improved DWTSVD Based Robust Digital Image Watermarking for Color Image. (2017) " International Journal of Engineering and Manufacturing (IJEM) (MECS) 7, no. 1: 49-59.

[21] Gökçen Çetinel, LLukman Çerkezi. "Robust Chaotic Digital Image Watermarking Scheme based on RDWT and SVD. (2016) " International Journal of Image, Graphics and Signal Processing (MECS) 8, no. 8: 58-67.

[22] Gunjal, Baisa L. "Robust, Secure and High Capacity Watermarking Technique based on Image PartitioningMerging Scheme. (2016) " International Journal of
Information Technology and Computer Science (MECS) 8, no. 4: 74-85.

[23] Kaiser J. Giri, Mushtaq Ahmad Peer, P. Nagabhushan. (2015) "A Robust Color Image Watermarking Scheme Using Discrete Wavelet Transformation." International Journal of Image, Graphics and Signal Processing (MECS) 7, no. 1: 47-52.

\section{Authors' Profiles}

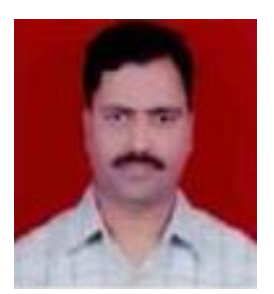

Dr. Dayanand G. Savakar ( Date of birth 02-10-1968), having 26 years of teaching and 12 years of research experience, he has completed his B.E degree from Karnataka University Dharwad, Post Graduation from Birla Institute of Technology and Ph.D. from Visvesvaraya Technological University., Belgaum, India. He is now working as Associate Professor, in the Department of Computer Science, Post Graduate Centre, Rani Channamma University, Vijayapur-Karnataka, India. He has published more than 35 research articles in international journals /conferences. Currently he is guiding eight Ph.D candidates. He served/serving as a member of various boards of several universities. His areas of interests are - Image Processing, Pattern recognition and Information security.

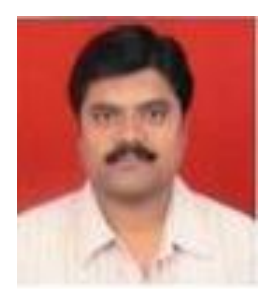

Mr. Anand Ghuli (Date of birth 17-041978), having 12 years of teaching and 5 years of research experience, he has completed B.Sc degree from Karnataka University Dharwad, MCA Post Graduation from Visvesvaraya Technological University and pursuing $\mathrm{Ph} . \mathrm{D}$ from Visvesvaraya Technological University., Belagavi, India. He is now working as Assistant Professor in the Department of Computer Applications (MCA), V.P.Dr. P.G.Halakatti College of Engineering and Technology, Vijayapur-Karnataka, India. His areas of interests are Information security and Image Processing.

How to cite this paper: Dayanand.G.Savakar, Anand Ghuli," Serial Digital Color Image Watermarking using Composite Scheme", International Journal of Image, Graphics and Signal Processing(IJIGSP), Vol.9, No.11, pp. 48-61, 2017.DOI: $10.5815 /$ ijigsp.2017.11.06 